\title{
Almbiente Contábil
}

\section{REVISTA AMBIENTE CONTÁBIL}

Universidade Federal do Rio Grande do Norte

ISSN 2176-9036

Vol. 10. n. 1, jan./jun. 2018

Sítios: http://www.periodicos.ufrn.br/ambiente http://ccsa.ufrn.br/ojs/index.php?journal=contabil http://www.atena.org.br/revista/ojs-2.2.3-06/index.php/Ambiente

Artigo recebido em: 17.03.2017. Revisado por pares em: 21.11.2017. Reformulado em: 18.12.2017. Avaliado pelo sistema double blind review.

SATISFAÇÃO DOS ALUNOS COM O CURSO DE CIÊNCIAS CONTÁBEIS: UMA ANÁLISE EM DIFERENTES INSTITUIÇÕES DE ENSINO SUPERIOR

STUDENTS SATISFACTION WITH THE COURSE OF ACCOUNTING: AN ANALYSIS IN DIFFERENT HIGHER EDUCATION INSTITUTIONS

\section{SATISFACCIÓN DE LOS ALUMNOS COM EL CURSO DE CIENCIAS CONTABLES: UM ANÁLISIS EN DIFERENTES INSTITUCIONES DE ENSEÑANZA SUPERIOR}

Autores

Suzete Antonieta Lizote

Doutora em Administração e Turismo - Professora do Programa de Pós-graduação em Administração - PPGA e do Curso de Ciências Contábeis da Universidade do Vale do Itajaí Univali. Endereço: Avenida Marcos Konder, 1100 - Apto 801 - Itajaí/SC - 88301-302 -

Telefone: 047.99114.6748. Identificadores (ID): Lattes: http://lattes.cnpq.br/0950927927432713

E-mail: lizote@univali.br

Miguel Angel Verdinelli

Doutor em Oceanografia pela Universidade de São Paulo (USP) - São Paulo - Professor do Programa de Pós-graduação em Administração - PPGA da Universidade do Vale do Itajaí Univali. Endereço: Rua Dr. Francisco Rangel, 5, Bairro Fazenda, Itajaí - SC - 88302.662 -

Telefone: 047. 99165,4357 Identificadores (ID): Lattes: http://lattes.cnpq.br/5991426676776328 Research Gate: https://www.researchgate.net/profile/Miguel_Verdinelli

E-mail:nupad@univali.br

José Carlos Terres

Mestre em Contabilidade - Coordenador e Professor do Curso de Graduação em Ciências Contábeis - Univali/ Itajaí/SC. Endereço: Avenida Marcos Konder, 1100 - Apto 801 Itajaí/SC - 88301-302 - Telefone: 047.99101.5640 Identificadores (ID): Lattes: http://lattes.cnpq.br/4721485514197028 
Suzete Antonieta Lizote, Miguel Angel Verdinelli, José Carlos Terres, Elen Sauer Camozzato e Jacqueline dos Santos Seemann

Elen Sauer Camozzato
Mestre em Administração - PPGA da Universidade do Vale do Itajaí - Univali/Biguaçu/SC
Rua Hildebrando Westphalen, 110 - Palmeira das Missões - RS - 98300.000 - Telefone:
54.9626 .1409$.
Identificadores (ID):
Lattes: http://lattes.cnpq.br/1072623053946671
Research Gate: https://www.researchgate.net/profile/Elen_Sauer_Camozzato
E-mail: elen.adm12@ 9 hotmail.com

Jacqueline dos Santos Seemann Mestre em Administração pela Univali/Itajaí/SC - Professora do Curso de Marketing Univali/ Balneário Camboriú/SC. Endereço: Rua Imbituba, 248 - Itajaí - SC - 88303.570 -

Telefone: 047.99940.3001.

Identificadores (ID):

Lattes: http://lattes.cnpq.br/7046408313762904

E-mail: jaquelineseemann@ @otmail.com

\begin{abstract}
RESUMO
O objetivo deste estudo foi avaliar como o interesse do estudante (IE) e o envolvimento do professor (EP) influenciam na satisfação geral (SG) dos alunos de graduação em Ciências Contábeis em diferentes tipos de Instituições de Ensino Superior (IES). Para tanto se levantaram dados em duas universidades, uma pública e gratuita e outra comunitária e de ensino pago, e num centro universitário privado e com finalidade lucrativa. Os dados foram obtidos com um questionário respondido numa escala de concordância pelos alunos. Também se levantaram variáveis sociodemográficas para analisar a influência de outros fatores. No marco teórico se focalizou na qualidade dos serviços educacionais e na satisfação que eles produzem. Os procedimentos metodológicos incluíram comparações de médias e análise de regressão a partir dos escores fatoriais gerados para cada constructo. Os resultados obtidos permitem concluir que o gênero não influencia na satisfação, mas os estudantes dos períodos iniciais mostram-se mais satisfeitos. Por sua vez, o envolvimento do professor e o interesse do estudante têm influencia sobre a satisfação geral, contudo de maneira diferente. No centro universitário EP e IE apresentam um poder explicativo maior da SG, embora a satisfação nesse tipo de IES seja estatisticamente menor que nas duas universidades. As implicâncias práticas destes achados podem contribuir para uma melhor adequação do currículo do curso e dos planos de ensino dos docentes.
\end{abstract}

Palavras-chave: Satisfação. Estudantes. Ciências Contábeis.

ABSTRACT
The objective of this study was to evaluate how student interest (IE) and teacher involvement
(EP) influence the general satisfaction (SG) of undergraduate students in Accounting in
different types of Higher Education Institutions (HEIs). For this purpose data were collected
in two universities, one public and free of charge and other community and paid education,
and in a private and for-profit university center. The data were obtained with a questionnaire
answered on a scale of agreement by the students. Sociodemographic variables were also
taken to analyze the influence of other factors. The theoretical framework focused on the
quality of educational services and the satisfaction they produce. The methodological

Revista Ambiente Contábil - ISSN 2176-9036 - UFRN - Natal-RN. v. 10. n. 1, p. 293 - 307, jan./jun. 2018. 
Suzete Antonieta Lizote, Miguel Angel Verdinelli, José Carlos Terres, Elen Sauer Camozzato e Jacqueline dos Santos Seemann

procedures included mean comparisons and regression analysis from the factor scores generated for each construct. The results obtained allow concluding that gender does not influence satisfaction, but students from the initial semesters are more satisfied. In turn, teacher involvement and student interest influence general satisfaction, however differently. In the university center EP and IE present a greater explanatory power of the SG, although the satisfaction in this type of HEI is statistically smaller than in the two universities. The practical implications of these findings may contribute to a better adaptation of the curriculum of the course and of the teaching plans.

Keywords: Satisfaction. Students. Accounting Sciences.

\section{RESUMEN}

El objetivo de este estudio fue evaluar como el interés del estudiante (IE) y el envolvimiento del profesor (EP) influyen en la satisfacción general (SG) de los alumnos de grado en Ciencias Contables de diferentes tipos de Instituciones de Enseñanza Superior (IES). Para ello se levantaron datos en dos universidades, una pública y gratuita y otra comunitaria y de enseñanza paga, así como en un centro universitario privado con finalidad lucrativa. Los datos fueron obtenidos con un cuestionario respondido por los alumnos en una escala de concordancia. También se registraron variables sociodemográficas para analizar la influencia de otros factores. En el marco teórico se focalizó en la calidad de los servicios educacionales y en la satisfacción que ellos producen. Los procedimientos metodológicos incluyeron comparaciones de medias y análisis de regresión a partir de los escores factoriales generados para cada constructo. Los resultados alcanzados permiten concluir que el género no influye en la satisfacción, pero los estudiantes de los períodos iniciales se muestran más satisfechos. Por su vez, el envolvimiento del profesor y el interés del estudiante tienen influencia sobre la satisfacción general, con todo de manera diferente. En el centro universitario EP e IE presentan un poder explicativo mayor de la SG, aunque la satisfacción en ese tipo de IES sea estadísticamente menor que en las dos universidades. Las implicaciones prácticas de estas conclusiones pueden contribuir para una mejor adecuación del curriculum del curso y de los planos de enseñanza de los docentes.

Palabras claves: Satisfacción. Estudiantes. Ciencias Contables.

\section{INTRODUÇÃO}

A oferta de serviços educacionais no Brasil tem aumentado notoriamente após a promulgação da Lei de Diretrizes e Bases da Educação Nacional (Lei $n^{\circ}$ 9394/96) e isto ocasionou uma expressiva concorrência entre as Instituições de Ensino Superior (IES), em particular nos cursos de graduação e pós-graduação lato sensu. Entretanto, as exigências do Ministério da Educação quanto às adaptações dos projetos pedagógicos, qualificação do corpo docente e infraestrutura da IES, têm obrigado que as mesmas atingissem níveis cada vez mais elevados de eficiência em sua gestão, tendo como objetivo fundamental a satisfação dos seus alunos.

Segundo pontuam Coda e Silva (2004) a satisfação com os cursos universitários envolve $\mathrm{o}$ atendimento das expectativas dos acadêmicos, sendo uma das condições determinantes da mesma a qualidade que eles possuam. Então, por causa das alternativas disponíveis no mercado de serviços de ensino superior, a própria sobrevivência das instituições pode estar afetada se a qualidade do serviço prestado não for aquela esperada.

Diversas pesquisas brasileiras têm mostrado que a satisfação dos estudantes é de grande importância para o sucesso das IES e um referente adequado da qualidade dos serviços (GONÇALVES FILHO, GUERRA; MOURA, 2003; CODA; SILVA, 2004; VIEIRA, 
Suzete Antonieta Lizote, Miguel Angel Verdinelli, José Carlos Terres, Elen Sauer Camozzato e Jacqueline dos Santos Seemann

MILACH; HUPPES, 2008; VERDINELLI, SOUZA; TOMIO, 2009; LIZOTE, VERDINELLI; LANA, 2011; LIZOTE, LANA; VERDINELLI, 2013). De igual maneira, para outros tipos de organizações verifica-se que um elevado nível de qualidade nos serviços prestados oferece benefícios significativos, em termos de melhorias na participação de mercado, produtividade e motivação, dentre outros.

No intuito de reter e captar alunos algumas IES medem internamente a satisfação dos alunos e dos professores, assim como a qualidade de seus serviços. Uma das finalidades do acompanhamento da satisfação dos discentes é identificar suas expectativas (SCOTT, 1999), para assim aumentar tanto a taxa de retenção quanto a lealdade dos alunos. Para tanto é fundamental diminuir as diferenças entre o desejado e o experienciado.

Os modelos mais atualizados de satisfação dos clientes tratam essa variável desde uma perspectiva dinâmica (WALTER, TONTINI; DOMINGUES, 2005) ao concebê-la como um processo que involucra os atos de comprar, usar e vender. Esta perspectiva distingue que a reação psicológica do cliente para um serviço não pode ser considerada apenas como o resultado de um evento, mas como uma série de atividades e reações contínuas ao longo do tempo. Confirma-se, portanto a necessidade de prestar os serviços de modo a evidenciar o maior número possível de atributos a serem percebidos pelos clientes. Há um entendimento cada vez maior de que as IES devem reforçar a qualidade de seus serviços e, assim procedendo, agregar valor aos seus estudantes. O que se torna um diferencial competitivo relevante.

No Brasil, baseado no modelo de Paswan e Yong (2002), Vieira; Milach e Huppes (2008) analisaram a satisfação dos alunos de ciências contábeis de uma universidade pública. Empregando esse mesmo modelo, Lizote et al. (2014) realizaram um estudo com estudantes de ciências contábeis de duas instituições de ensino superior privadas. Das oito hipóteses elencadas pelos autores em sua pesquisa sete foram confirmadas, sendo apenas uma, a que relaciona de maneira negativa a exigência do curso com o interesse do aluno, a que não teve significância para ser comprovada.

Considerando os conhecimentos já existentes quanto à satisfação dos alunos de graduação em ciências contábeis percebe-se que não se efetuaram comparações entre IES de ensino gratuito ou pago, bem como entre universidades públicas e comunitárias. Neste sentido, emerge o seguinte problema de pesquisa: o interesse do estudante e o envolvimento de professor influenciam de modo semelhante na satisfação geral dos alunos de graduação em Ciências Contábeis em diferentes tipos de instituições de ensino superior?

Procurando responder àquele questionamento, estabeleceu-se como o objetivo geral do presente estudo avaliar como o interesse do estudante e o envolvimento do professor influenciam na satisfação geral dos alunos de graduação em Ciências Contábeis em diferentes tipos de Instituições de Ensino Superior. A pesquisa foi realizada em duas universidades (pública gratuita e comunitária de ensino pago) e um centro universitário de regime privado lucrativo.

Com o presente estudo busca-se obter evidências empíricas respeito das relações que se esperam entre os construtos analisados com base nos conceitos teóricos arrolados como marco da pesquisa. Por outro lado, salienta-se que o sucesso de uma instituição de ensino está diretamente ligado ao comprometimento e condição do corpo discente e à desenvoltura dos gestores e docentes. As implicâncias práticas destes achados podem contribuir para uma melhor adequação do currículo do curso e dos planos de ensino dos docentes.

O presente estudo encontra-se estruturado em 5 seções, iniciando com a presente introdução. A segunda seção apresenta o marco teórico do artigo, enquanto se abordam na seção seguinte os procedimentos metodológicos. Na seção 4 estão evidenciadas as análises dos dados e os resultados, sendo que na sequência apresentam-se as considerações finais e sugestões para futuros trabalhos. Por fim, apresenta-se a listagem das referências citadas. 
Suzete Antonieta Lizote, Miguel Angel Verdinelli, José Carlos Terres, Elen Sauer Camozzato e Jacqueline dos Santos Seemann

\section{FUNDAMENTAÇÃO TEÓRICA}

Nesta seção se trata dos temas que fundamentam o trabalho, quais sejam: qualidade em serviços e satisfação de estudantes.

\subsection{QUALIDADE EM SERVIÇOS}

As empresas, independentemente do seu ramo de atividade, estão adaptando-se rapidamente para desenvolver uma gestão que atenda as expectativas e necessidades de seus clientes, pois os consumidores estão cada vez mais exigentes com os produtos e serviços que lhes são ofertados. O Brasil, em consonância com os paradigmas atuais, também se encontra envolto com essas preocupações. Segundo Alberton et al. (1999), os temas relacionados à qualidade tornaram-se mais evidentes no Brasil na década de 1990, quando diversas organizações começaram seus programas de qualidade total, incluindo as IES.

O setor de serviços, conforme destaca Holanda (2007), está se tornando a fonte básica de riqueza, comércio e crescimento econômico em todo o mundo. O que lhe confere uma importância cada vez maior no desenvolvimento das sociedades. Porém, de nada adianta se os serviços prestados não forem de qualidade, pois esse atributo é o fator que proporciona uma das maneiras de que seu prestador obtenha sucesso na fidelização dos clientes e se destaque entre os concorrentes.

Como prestadoras de serviços especializados, as IES devem envidar esforços em atender às necessidades e expectativas dos seus alunos. Além disso, a intensa concorrência nacional e internacional, magnificando as forças competitivas no mercado de ensino, estimula a adoção de estratégias de orientação para a diferenciação de seus produtos (TEMIZER; TURKYILMAZ, 2012). Em tal contexto, as universidades, ao ser organizações que prestam serviços educacionais à sociedade, não podem ficar alheias a essas exigências.

Conforme explica Finger (2000) as IES passam por um processo bem mais complexo que as outras organizações devido à natureza de seu negócio ser um serviço essencialmente intangível. Como resultado desse processo elas se envolvem em práticas educativas competitivas, mas apesar de alcançar algum grau de reconhecimento a qualidade do ensino superior, em geral, fica aquém de atingir um nível de excelência global (SENTHILKUMAR; ARULRAJ, 2011). Contudo, quando os alunos percebem uma boa qualidade na prestação do serviço educacional se potencializa a propensão a recomendar a instituição.

Nesta perspectiva, para atingir a qualidade deve-se efetuar uma adequada gestão institucional e avaliar como mensura-la (BROCHADO, 2009). Afinal, ao avaliar a qualidade monitoram-se também as estratégias adotadas, retratando a efetividade dos esforços dispendidos. Marchetti e Prado (2004) argumentam que a busca pela melhoria da qualidade na educação superior proporciona diversos ganhos para a sociedade como um todo, pois não basta formar e especializar mais profissionais é necessário que eles estejam preparados qualitativamente para atuar e se manter no mercado de trabalho.

Portanto, como Watty (2005) salienta, a vantagem competitiva de uma instituição depende da qualidade e do valor dos bens e serviços que oferece. Acrescenta também, que no contexto de serviços, a qualidade pode ser o fundamento do diferencial em relação à concorrência. Desta forma, é imperativo entregar um serviço superior às expectativas dos usuários, para assim, fortalecer sua posição no mercado, garantindo, também, a satisfação do cliente.

Diante deste tipo de perspectiva, Finger (2000) já comentava que é necessário entender o que constitui qualidade do ponto de vista do cliente, realizar o que é necessário para satisfazê-lo e ir além de suas expectativas. Atender as demandas exigidas pelos clientes constitui a estratégia para oferta de serviços excelentes, adequados aos ditames do mercado, 
Suzete Antonieta Lizote, Miguel Angel Verdinelli, José Carlos Terres, Elen Sauer Camozzato e Jacqueline dos Santos Seemann

que resultam e estimulam seu reuso. Com isto, a busca pela qualidade nas Instituições de Ensino Superior passa pela descoberta das necessidades dos alunos, procurando melhorar os padrões de qualidade e, consequentemente, a satisfação dos mesmos, para assim prosseguir com o ciclo de crescimento e permanência no mercado. Ao considerar que a qualidade de um serviço está condicionada ao sentimento (positivo ou negativo) de atendimento das expectativas de um cliente é relevante conhecer as principais características da satisfação.

\subsection{SATISFAÇÃO DE ESTUDANTES}

A concorrência estimula às organizações a inovar seus produtos e serviços, tendo como foco o atendimento às necessidades do consumidor. Conforme colocava McKenna (1992), o cliente é quem determina o que é uma organização. Enfatiza também, que para a empresa o decisivo é o que pensa o cliente a seu respeito, ou seja, para que a organização desenvolva um trabalho que atenda as expectativas de sua clientela é imperativo conhecer suas verdadeiras necessidades e desejos. Segundo Kotler (1998), a satisfação é o sentimento de desapontamento ou prazer resultante da comparação do desempenho esperado de um produto ou serviço em relação às expectativas da pessoa. Sendo assim, no campo da educação é necessário observar atentamente alguns itens essencialmente importantes para o ensino superior. Mezomo (1997) enfatiza que as IES devem almejar a qualidade de forma constante e determinada e que uma medida de satisfação dos discentes deve ser adotada como a ferramenta fundamental no processo gerencial e no de ensino-aprendizagem.

Torna-se importante ressaltar que as universidades brasileiras, há alguns anos, atuavam de forma passiva nas questões educacionais na sua relação com o mercado. Porém, em função da concorrência, conforme destacam Gonçalves Filho et al. (2003), atualmente necessitam ser proativas nas suas ações estratégicas. Em especial na identificação das necessidades e expectativas de um mercado cada vez mais exigente e seletivo. Complementam Gruber et al. (2010) ao dizer que as Instituições de Ensino Superior devem manter-se permanentemente atualizadas, com estrutura adequada e docentes capacitados para assim formar profissionais habilitados para atuarem no mercado.

Partindo do pressuposto que o sucesso de uma instituição de ensino está diretamente ligado ao comprometimento e condição do corpo discente e à desenvoltura dos gestores e docentes, observa-se que a satisfação estudantil é uma resposta afetiva por um período de tempo, que resulta da avaliação dos serviços pedagógicos e de apoio aos estudos ofertados aos discentes (PALÁCIO; MENESES; PÉREZ, 2002). Para Navarro; Iglesias e Torres (2005), o conceito de satisfação é uma variável de administração, essencial para alcançar os objetivos estratégicos de instituições universitárias. Pesquisas em diferentes áreas do saber e IES confirmam o valor do envolvimento do professor com o curso, por exemplo, dentre outras, as de Paswan e Yong (2002), Palacio et al. (2002), Bortolotti et al. (2004), Navarro et al. (2005) e Vieira et al. (2008).

Alunos satisfeitos com os serviços internos da IES e com os cursos que ela oferece influenciam positivamente na percepção que a sociedade e futuros alunos têm a seu respeito, aumentando a demanda. Por outro lado, a percepção negativa terá efeito contrário (GUTIÉRREZ; CAMBLOR, 2007). Por sua parte, Porturak (2014) assinala que identificar como os diferentes atributos das IES afetam a satisfação dos discentes torna-se crítico para um gerenciamento eficaz. Nesta linha de pensamento, Awan e Rehman (2013) resaltam que a IES que busca ser reconhecida como uma instituição de referência, notada pela qualidade de suas ações e resultados, tem na satisfação de seus clientes internos (os alunos) e externos (a sociedade) um dos seus principais valores. Em geral, esses constructos trabalham-se de maneira separada. Na área de ciências contábeis são exemplo disto, dentre outras, a pesquisa de Leite e Guimarães (2004) e de Watty (2005) para qualidade e para satisfação as de De Faria

Revista Ambiente Contábil - ISSN 2176-9036 - UFRN - Natal-RN. v. 10. n. 1, p. 293 - 307, jan./jun. 2018. 
Suzete Antonieta Lizote, Miguel Angel Verdinelli, José Carlos Terres, Elen Sauer Camozzato e Jacqueline dos Santos Seemann

et al. (2006), Lagioia et al. (2007), Vieira et al. (2008), Lizote et al. (2012) e a de Gomes et al. (2013).

A satisfação dos estudantes é determinada por vários fatores, dentre os quais Paswan e Young (2002), destacam cinco: a) envolvimento do professor; b) interesse do aluno; c) interação professor-aluno; d) exigência do curso; e, e) organização do curso. Esses mesmos construtos foram utilizados por Vieira; Milach e Huppes (2008) no seu estudo com alunos do curso de Ciências Contábeis de uma universidade pública empregando a modelagem em equações estruturais. Para avaliar os relacionamentos os autores estabeleceram oito hipóteses.

No presente estudo, desenvolvido com acadêmicos de três Instituições de Ensino Superior, das quais duas são universidades (pública gratuita e comunitária de ensino pago) e um centro universitário de regime privado lucrativo, a satisfação foi mensurada por uma nota de autoavaliação e também pelo questionário descrito no estudo de Verdinelli; Souza e Tomio (2009).

\section{PROCEDIMENTOS METODOLÓGICOS}

Para efetuar a pesquisa o levantamento do material foi realizado por meio de um questionário de autopreenchimento aplicado no primeiro semestre de 2016 aos alunos dos cursos de Ciências Contábeis de três Instituições de Ensino Superior (IES), uma universidade comunitária do Estado de Santa Catarina e uma universidade pública e um centro universitário do Estado de Amazonas. O instrumento de coleta de dados esteve composto por 23 asseverações a serem respondidas através de uma escala Likert de concordância de 5 pontos, indo desde discordo totalmente (1) até concordo plenamente (5).

O questionário compreendia três blocos, referidos respectivamente aos constructos envolvimento do professor (EP) com 7 itens; interesse do estudante (IE), com 6 itens; e, satisfação geral (SG), com 10 itens. Além desses dados se solicitava aos alunos indicar o gênero, em que período estava matriculado e qual era sua satisfação medida por uma nota entre 0 e 10.

Os dados obtidos foram digitados numa planilha eletrônica, onde inicialmente se fez o pré-processamento dos mesmos segundo as indicações de Hair Jr. et al. (2009). Observou-se que existiam 14 dados faltantes nos 163 questionários recebidos na universidade comunitária, mas como não foi reconhecido nenhum padrão foram preenchidos com o valor da mediana do item considerado. Como os dados levantados em Amazonas foram mais numerosos se excluíram os questionários que tinham dados faltantes, computando-se 223 da universidade pública e 269 do centro universitário. A seguir, avaliaram-se os outliers com a função gráfica Box-Plot, com a que foram reconhecidos nas três bases 193 ao total. Como não estavam distribuídos seguindo algum padrão optou-se por mantê-los. Não se registraram erros de digitação. Por fim, e com a finalidade de avaliar a normalidade da distribuição das variáveis considerando que os dados provinham de escalas tipo Likert, efetuaram-se os cálculos da assimetria e curtose (HAIR Jr. et al., 2009). Finney e DiStefano (2006) afirmam que dados com coeficientes de até 2 de assimetria e até 7 de curtose, em módulo, podem ser considerados quase normais.

Os métodos estatísticos empregados para comparar as médias das notas atribuídas à satisfação foram o teste $\mathrm{t}$ e a análise de variância. As Anovas realizadas foram sempre univariadas e, portanto, muito robustas às violações da normalidade e da homocedasticidade (HARRIS, 1975). No caso de existirem diferenças na comparação simultânea deu-se prosseguimento com o teste pareado a posteriori de Scheffe. Os métodos multivariados usados foram análise fatorial (AF) e regressão múltipla (RM). Na $\mathrm{AF}$ os fatores foram extraídos pelo método de componentes principais e definiu-se a unidimensionalidade segundo o critério de Kaiser. Os itens considerados deviam ter uma correlação de no mínimo 0,7 com o fator, uma comunalidade igual ou maior do que 0,5 e a variância extraída maior que $50 \%$. 
Suzete Antonieta Lizote, Miguel Angel Verdinelli, José Carlos Terres, Elen Sauer Camozzato e Jacqueline dos Santos Seemann

Antes de realizar a AF foi calculado o coeficiente alfa de Cronbach para cada constructo considerado e a correlação do item com o total, conforme o procedimento sugerido por Churchill Jr. (1979). Posteriormente utilizaram-se o teste de Kaiser, Olkin e Meyer (KMO), o de Bartlett e a medida de adequação da amostra (MSA) para confirmar a factibilidade de empregar a análise fatorial. Nessas condições se geraram os escores fatoriais com os quais se efetuou a RM. Segundo Tabachnick e Fidel (2001), a AF pode ser utilizada para criar variáveis independentes ou dependentes que podem ser utilizadas posteriormente em modelos de regressão.

\section{RESULTADOS}

Conforme apresentado anteriormente, a amostra obtida totalizou 665 questionários válidos, tendo a satisfação geral uma média de 7,67 com desvio-padrão em 1,53. Esses valores para os alunos do sexo masculino foram de 7,64 e 1,50 e para as alunas 7,69 e 1,55, respectivamente. Ao realizar um teste t se verifica que entre esses valores não há diferenças significativas. Para todas as IES em conjunto o valor mínimo computado foi 0 e o máximo 10. Entretanto a nota máxima foi dada por alunos das três IES enquanto a mínima somente pelos estudantes do centro universitário. Para estarem representadas as três instituições o valor menor foi 4. Incluindo todos os respondentes que pontuaram com valores iguais ou menores a 4 eles representam apenas $3,2 \%$ e os que se atribuíram notas de satisfação 9 e 10 foram $26,4 \%$.

Se comparadas as médias gerais das três instituições os valores obtidos com os dados das universidades foram estatisticamente iguais entre si, 7,96 para a pública e 7,94 para a comunitária, e maiores do que a nota 7,28 de satisfação média do centro universitário $(\mathrm{p}<0,001)$. Feita a ANOVA para saber se o período em que o aluno está matriculado influencia sobre a satisfação geral expressa pela nota de autoavaliação que eles se atribuíram verificou-se que há diferença na comparação simultânea.

Ao efetuar o teste de Scheffe para as comparações a posteriori confirmou-se que os alunos do primeiro período pontuam de modo mais elevado sua satisfação, atingindo uma média de 8,46 , que foi significativamente maior do que a média 7,49 dos terceiros períodos $(\mathrm{p}=0.04)$ e 7,40 dos sextos períodos $(\mathrm{p}=0,03)$.

Avaliada a assimetria e a curtose, com exceção do valor de assimetria computado para a questão 8, que foi de $-2,03$ para o centro universitário, os demais valores encontram-se dentro dos limites estabelecidos por Finney e DiStefano (2006) para que as variáveis sejam consideradas quase-normais. Devido a que a diferença com o limite é de apenas três centésimos optou-se por manter esse item na base de dados.

Com cada constructo fez-se a correlação item-total e se calculou a confiabilidade com o alfa de Cronbach, conforme o sugerido por Churchill Jr. (1979). Os resultados, expostos na Tabela 1, indicam que para todos os constructos as estimações do alfa de Cronbach sempre foram maiores do que o valor recomendado de 0,7 .

Por sua vez, os valores da correlação item-total foram maiores do que 0,4 e a correlação inter itens só teve três valores menores a 0,4 registrado para as universidades. 
Suzete Antonieta Lizote, Miguel Angel Verdinelli, José Carlos Terres, Elen Sauer Camozzato e Jacqueline dos Santos Seemann

\section{Tabela 1 - Indicadores de factibilidade de efetuar uma análise fatorial com os constructos considerados.}

\begin{tabular}{|c|c|c|c|c|c|c|}
\hline \multirow{2}{*}{ IES } & \multirow{2}{*}{ CONSTRUCTOS } & \multirow{2}{*}{$\begin{array}{c}\alpha \text { de } \\
\text { Cronbach }\end{array}$} & \multicolumn{2}{|c|}{ CORRELAÇÃO } & \multirow{2}{*}{ K-M-O } & \multirow{2}{*}{ M S A } \\
\hline & & & inter-itens & item-total & & \\
\hline \multirow{3}{*}{ 离 } & $\begin{array}{l}\text { Envolvimento do } \\
\text { Professor }\end{array}$ & 0,809 & 0,385 & $>0,45$ & 0,833 & $>0,749$ \\
\hline & Interesse do Estudante & 0,769 & 0,376 & $>0,40$ & 0,787 & $>0,765$ \\
\hline & Satisfação geral & 0,876 & 0,420 & $>0,40$ & 0,88 & $>0,747$ \\
\hline \multirow{3}{*}{ 忘 } & $\begin{array}{l}\text { Envolvimento do } \\
\text { Professor }\end{array}$ & 0,758 & 0.314 & $>0,40$ & 0,751 & $>0,724$ \\
\hline & Interesse do Estudante & 0,812 & 0,441 & $>0,40$ & 0,813 & $>0,752$ \\
\hline & Satisfação geral & 0,881 & 0,437 & $>0,40$ & 0,877 & $>0,790$ \\
\hline \multirow{3}{*}{ 党: } & $\begin{array}{l}\text { Envolvimento do } \\
\text { Professor } \\
\end{array}$ & 0,916 & 0,613 & $>0,65$ & 0,919 & $>0,895$ \\
\hline & \begin{tabular}{|l|} 
Interesse do Estudante \\
\end{tabular} & 0,871 & 0,540 & $>0,55$ & 0,855 & $>0,817$ \\
\hline & Satisfação geral & 0,916 & 0,531 & $>0,40$ & 0,914 & $>0822$ \\
\hline
\end{tabular}

Fonte: Dados da pesquisa.

Os testes de Kaiser, Meyer e Olkin foram significativos para todos os constructos, superando sempre o valor sugerido de 0,7 . De igual maneira, os valores calculados da medida de adequação da amostra a partir da matriz de correlação anti-imagem superaram aquele valor em todos os constructos das três IES. Finalmente, ao realizar o teste de esfericidade de Bartlett os resultados foram todos significativos e indicaram que com os dados levantados para os constructos pode-se empregar a análise fatorial.

As análises fatoriais exploratórias efetuadas individualmente com cada constructo e de acordo com as restrições descritas nos procedimentos metodológicos resultaram na exclusão de alguns itens. Na Tabela 2 se apresentam os resultados alcançados.

Tabela 2 - Resumo da Análise Fatorial. Mínimos exigidos: * 3 variáveis; ** valores $>1,00 ; * * *$ valores $>0,50$.

\begin{tabular}{|c|c|c|c|c|c|}
\hline IES & CONSTRUCTOS & $\begin{array}{l}\text { Número } \\
\text { inicial de } \\
\text { variáveis }\end{array}$ & $\begin{array}{c}\text { Número } \\
\text { Final de } \\
\text { variáveis * }\end{array}$ & Autovalores** & $\begin{array}{c}\text { Variância } \\
\text { extraída } * * *\end{array}$ \\
\hline \multirow{3}{*}{ 离异 } & Envolvimento do Professor & 7 & 4 & 2,663 & 0,5326 \\
\hline & Interesse do Estudante & 6 & 3 & 2,051 & 0,6837 \\
\hline & Satisfação geral & 10 & 6 & 3,847 & 0,6412 \\
\hline \multirow{3}{*}{ ن } & Envolvimento do Professor & 7 & 3 & 1,817 & 0,6056 \\
\hline & Interesse do Estudante & 6 & 3 & 2,03 & 0,6767 \\
\hline & Satisfação geral & 10 & 6 & 3,712 & 0,6187 \\
\hline \multirow{3}{*}{ 党 } & Envolvimento do Professor & 7 & 7 & 4,668 & 0,6668 \\
\hline & Interesse do Estudante & 6 & 6 & 3,684 & 0.6140 \\
\hline & Satisfação geral & 10 & 8 & 5,211 & 0,6514 \\
\hline
\end{tabular}

Fonte: Dados da pesquisa.

Na primeira análise, feita com os dados da universidade pública, se verifica que a RM foi significativa, conforme indica a análise de variância da regressão $(p<0,000)$ e a significância dos coeficientes angulares do EP e o IE. Por sua parte, o coeficiente de determinação ajustado mostra que aproximadamente $44,5 \%$ da satisfação geral do aluno pode 
Suzete Antonieta Lizote, Miguel Angel Verdinelli, José Carlos Terres, Elen Sauer Camozzato e Jacqueline dos Santos Seemann

ser estimada pelo efeito do envolvimento do professor e o interesse do estudante, como se exibe na Tabela 3.

Tabela 3 - Regressão múltipla para a satisfação geral (SG) estimada pelo envolvimento do professor (EP) e o interesse do estudante (IE). Universidade Pública.

\begin{tabular}{|c|c|c|c|c|c|c|}
\hline \multicolumn{7}{|c|}{$\begin{array}{l}\text { Variável dependente: } S G \text { (Uni. Pública) } \\
R=0,67080091 \mathrm{R}^{2}=0,44997386 \mathrm{R}^{2} \text { Ajustado }=0,44497363 \\
\mathrm{~F}(2,220)=89,990 \mathrm{p}<0,0000 \text { Erro padrão da estimativa: } 0,74500\end{array}$} \\
\hline $\mathrm{N}=233$ & $\beta$ & d.p. & $\mathrm{b}$ & d.p. & $t(220)$ & sig. \\
\hline Intercepto & & & 0,000 & 0,050 & 0,000 & 1,000 \\
\hline EP & 0,599 & 0,054 & 0,599 & 0,054 & 11,066 & 0,000 \\
\hline IE & 0,150 & 0,054 & 0,150 & 0,054 & 2,780 & 0,006 \\
\hline
\end{tabular}

Fonte: Dados da pesquisa.

Os resultados da regressão múltipla com os dados da universidade comunitária, apresentados na Tabela 4, mostram um alto valor $\mathrm{F}$ na Anova, confirmando que ela é significativa para estimar a satisfação a partir do envolvimento do professor e o interesse do estudante. Inclusive os coeficientes angulares de essas variáveis indicam que elas aportam um valor quase igual, de 0,4 por unidade de mudança, para a estimativa da SG. O R $\mathbf{2}^{\mathbf{2}}$ ajustado com um valor de 0,472 confirma que este modelo está mais bem ajustado que o da universidade pública.

Tabela 4 - Regressão múltipla para a satisfação geral (SG) estimada pelo envolvimento do professor (EP) e o interesse do estudante (IE). Universidade Comunitária.

\begin{tabular}{|c|c|c|c|c|c|c|}
\hline \multicolumn{7}{|c|}{$\begin{array}{l}\text { Variável dependente: SG (Univ. Comunitária) } \\
R=0,69173272 \mathrm{R}^{2}=0,47849416 \mathrm{R}^{2} \text { Ajustado }=0,47197533 \\
\mathrm{~F}(2,160)=73,402 \mathrm{p}<0,0000 \text { Erro padrão da estimativa: } 0,72665\end{array}$} \\
\hline $\mathrm{N}=163$ & $\beta$ & d.p. & $\mathrm{b}$ & d.p. & $t(220)$ & sig. \\
\hline Intercepto & & & 0,000 & 0,057 & 0,000 & 1,000 \\
\hline EP & 0,400 & 0,065 & 0,400 & 0,065 & 6,196 & 0,000 \\
\hline IE & 0,407 & 0,065 & 0,407 & 0,065 & 6,293 & 0,000 \\
\hline
\end{tabular}

Fonte: Dados da pesquisa.

O melhor ajuste do modelo de regressão se verifica para os dados do centro universitário, como é mostrado na Tabela 5. O valor da estatística $\mathrm{F}$ da Anova da regressão $(214,85)$ é mais do que o dobro dos calculados para as universidades, 89,990 e 73,402 respectivamente para a pública e a comunitária. Do mesmo modo, o valor do coeficiente de determinação ajustado é maior para o centro universitário, indicando que a SG pode ser estimada em um $61,5 \%$ pelas variáveis preditoras EP e IE. 
Suzete Antonieta Lizote, Miguel Angel Verdinelli, José Carlos Terres, Elen Sauer Camozzato e Jacqueline dos Santos Seemann

\section{Tabela 5 - Regressão múltipla para a satisfação geral (SG) estimada pelo envolvimento do professor (EP) e o interesse do estudante (IE). Centro Universitário.}

\begin{tabular}{|c|c|c|c|c|c|c|}
\hline \multicolumn{7}{|c|}{$\begin{array}{l}\text { Variável dependente: } S G \text { (Centro Universitário) } \\
\mathrm{R}=0,78591131 \mathrm{R}^{2}=0,61765658 \mathrm{R}^{2} \text { Ajustado }=0,61478182 \\
\mathrm{~F}(2,266)=214,85 \mathrm{p}<0,0000 \text { Erro padrão da estimativa: } 0,62066\end{array}$} \\
\hline $\mathrm{N}=269$ & $\beta$ & d.p. & $\mathrm{b}$ & d.p. & $\mathrm{t}(220)$ & sig. \\
\hline Intercepto & & & 0,000 & 0,038 & 0,000 & 1,000 \\
\hline EP & 0,655 & 0,050 & 0,655 & 0,050 & 13,173 & 0,000 \\
\hline IE & 0,182 & 0,050 & 0,182 & 0,050 & 3,664 & 0,000 \\
\hline
\end{tabular}

Fonte: Dados da pesquisa.

Cabe assinalar que as variáveis preditoras no centro universitário, e na universidade pública, têm uma participação diferente entre si. Em ambos os casos o envolvimento do professor é muito mais contributivo que o interesse do estudante, o que não acontece no caso da universidade comunitária que mostrou contribuições equivalentes.

\section{CONSIDERAÇÕES FINAIS}

No meio acadêmico as pesquisas relacionadas à aprendizagem e metodologias de ensino são frequentes. Através delas busca-se melhorar o nível de aprendizagem dos alunos e para tanto, se desenvolvem e implantam novas técnicas, métodos e modelos. Os estudos que consideram a qualidade do ensino em conjunto com a satisfação dos alunos com as práticas implantadas pelas instituições de ensino superior são escassos. No presente trabalho foi avaliado como o interesse do estudante e o envolvimento do professor influenciam na satisfação geral dos alunos de graduação em Ciências Contábeis em duas universidades, uma pública gratuita e outra comunitária de ensino pago, e um centro universitário de regime privado lucrativo.

Na revisão da literatura acerca do assunto confirma-se que é fundamental que as IES promovam pesquisas de satisfação junto a seus alunos. De tal maneira, poderão ser atendidas as necessidades e expectativas deles. Em particular na sua preparação e capacitação para a inserção no mercado de trabalho. Diversas são as formas de mensurar a satisfação e, como assinalam Paswan e Yong (2002) há vários fatores que influenciam. Dentre os que esses autores mencionam os mais relacionados diretamente são o envolvimento do professor e o interesse do estudante.

Depois da análise dos resultados deste estudo confirma-se também que a participação efetiva do professor no curso é de importância fundamental na determinação da satisfação do corpo discente. Entretanto, sua participação é diferente segundo o tipo de instituição considerada na pesquisa. Apenas na universidade comunitária existe equilíbrio entre o envolvimento do docente e o interesse do aluno.

Como se verificou para os três tipos de IES o interesse do estudante, embora possa ser menos influente, sempre foi significativo. Isto implica em que ambos os constructos estão relacionados com a satisfação dos alunos e sendo a satisfação relacionada com o aprendizado, logo fica clara sua importância. Portanto, os resultados obtidos permitem concluir que ao ser o envolvimento do professor, assim como os interesses do estudante determinantes da satisfação geral dos alunos com o curso, ambos os constructos devem ser levados em consideração pelas IES para melhor definir suas funções precípuas e seu marketing educacional.

Cabe salientar ainda como remarque conclusivo que não houve diferenças quanto à satisfação em relação ao gênero do aluno, mas sim quando se considerou o período em que o aluno está matriculado. Os mais satisfeitos são os alunos do primeiro período, o que deve ser 
Suzete Antonieta Lizote, Miguel Angel Verdinelli, José Carlos Terres, Elen Sauer Camozzato e Jacqueline dos Santos Seemann

um aspecto a levar em conta pelos gestores dos cursos.

Este estudo, com base nos resultados apresentados, apresenta como contribuição o entendimento de que aspectos como acessibilidade, atendimento, comprometimento dos alunos, capacitação dos docentes, comunicação, conteúdos abordados, metodologias, credibilidade, infraestrutura, entre outros, são indicadores de satisfação que podem ser utilizados pelas IES para avaliarem o desempenho de suas atividades, no intuito de melhorar os processos de aprendizagem relacionados aos aspectos de ensino, pesquisa, extensão, cidadania e cultura geral.

Sugere-se que próximas pesquisas sejam agregadas novos constructos para analisar em conjunto com a satisfação dos alunos. Em especial os aspectos referentes à qualidade do curso e do ensino, que se supõe tenham influência positiva na satisfação. Com tal esforço, acreditase que seja possível para as IES conhecerem melhor os estudantes e lhes oferecer serviços educacionais com qualidade e que satisfaçam suas expectativas, contribuindo também para o processo eficaz de ensino-aprendizagem.

\section{REFERÊNCIAS}

ALBERTON, L.; CARDOSO, O. R.; COSTA, J. I. P. Análise da implantação da qualidade total em uma instituição pública de educação. Florianópolis: Ed. UFSC, 1999.

AWAN, A. M.; REHMAN, M. A. Antecedents of higher degree students' satisfaction: a developing country perspective. Middle-East Journal of Scientific Research, v.18, n. 5, p. 651-659, 2013.

BORTOLOTTI, S. L. V.; PIÑERO VERDINELLI, M. E.; VERDINELLI, M. A. Avaliando a satisfação de alunos do ensino superior pelo curso que frequentam: um subsídio para a gestão universitária. In: Anais... IV Colóquio Internacional sobre Gestão Universitária na América do Sul. Florianópolis, 2004.

BROCHADO, A. Comparing alternative instruments to measure service quality in higher education. Quality Assurance in Education, v. 17, n. 2, p. 174-190, 2009.

CHURCHILL JR., G. A. A Paradigm for developing better measures of marketing constructs. Journal of Marketing Research, v. 16, p. 1, p. 64-74, 1979.

CODA, R.; SILVA, D. Sua escola de administração é uma excelente escola para se estudar? Descobrindo dimensões de alunos em cursos de administração: uma contribuição metodológica. In: Anais... XXVIII Encontro Nacional dos Programas de Pós-Graduação em Administração, Curitiba, 2004.

DE FARIA, A. C.; de COME, E.; POLI, J.; FELIPE, Y. X. O grau de satisfação dos alunos do curso de ciências contábeis: busca e sustentação da vantagem competitiva de uma IES privada. Enfoque: Reflexão Contábil [online], v. 25, p. 25-36, 2006.

FINGER, A. B. A qualidade dos cursos de mestrado em administração: uma avaliação pela percepção discente. 2000. Dissertação (Mestrado em Administração) - Universidade Federal de Santa Catarina, Florianópolis, 2000. 
Suzete Antonieta Lizote, Miguel Angel Verdinelli, José Carlos Terres, Elen Sauer Camozzato e Jacqueline dos Santos Seemann

FINNEY, S. J.; DiSTEFANO, C. Non-normal and categorical data in structural equation modeling. In: HANCOK, G. R.; MUELEER, R. O. Structural equation modeling: a second course. Charlotte: Information Age Publ., 2006.

GOMES, G.; DAGOSTINI, L.; CUNHA, P. R. Satisfação dos estudantes do curso de ciências contábeis: estudo em uma faculdade do Paraná. ReFAE - Revista da Faculdade de Administração e Economia, v. 4, p. 2, p. 102-123, 2013.

GONÇALVES FILHO, C.; GUERRA, R. S.; MOURA, A. Mensuração de satisfação, qualidade, lealdade, valor e expectativa em instituições de ensino superior: um estudo do modelo ACSI através de equações estruturais. In: Anais... XXVII Encontro Nacional dos Programas de Pós-Graduação em Administração. Atibaia, 2003.

GRUBER, T.; FU $\beta$, S.; VOSS, R.; GLÄSER-ZIKUDA, M. Examining student satisfaction with higher education services. Using a new measurement tool. International Journal of Public Sector Management, v. 23, n. 2, p. 105-123, 2010.

GUTIÉRREZ, S. A.; CAMBLOR, M. P. Principales factores de satisfacción entre los estudiantes universitarios. La unidad académica multidiciplinaria de agronomía y ciencias de la UAT. Revista Internacional de Ciencias Sociales y Humanidades, v. 8, n. 1, p. 163-192, 2007.

HAIR JR., J. F.; BLACK, W. C.; BABIN, B. J.; ANDERSON, R. E.; TATHAM R. L. Análise multivariada de dados. 5 Ed., Porto Alegre: Bookman, 2009.

HARRIS, R. J. A primer of multivariate statistics. New York: Academic Press, 1975.

HOLANDA, J. O desafio é ser atraente. Revista Ensino Superior, v. 100, p. 28-35, 2007.

KOTLER, P. Administração de marketing. 5 Ed., São Paulo: Atlas, 1998.

LAGIOIA, U. C. T.; SANTIAGO, H. L. F.; GOMES, R. B.; RIBEIRO FILHO, J. F. Uma investigação sobre as expectativas dos estudantes e o seu grau de satisfação em relação ao curso de Ciências Contábeis. Revista Contemporânea de Contabilidade, v. 1, n. 8, p. 121$138,2007$.

LEITE, C. E. B; GUIMARÃES, G. Qualidade nos cursos de ciências contábeis. Contabilidade Vista \& Revista, v. 15, n. 1, p. 35-51, 2004.

LIZOTE, S. A.; GABRIEL, A. V.; STEIN, F.; SERPA, G. V.; SILVA, M. J.; RODRIGUES, T. Satisfação dos alunos de Ciências Contábeis: um estudo em uma instituição de ensino superior comunitária. Revista Brasileira de Contabilidade, v. 201, p. 45-55, 2012.

LIZOTE, S. A.; LANA, J.; VERDINELLI, M. A. The influence of the gender in post-graduate student's satisfaction: evidences from a Brazilian university. In: Proceeding of European Academy of Management - EURAM, 2013.

LIZOTE, S. A.; VERDINELLI, M. A.; BORBA, J. A.; BRASIL, M. L. A. V. Satisfação dos acadêmicos com o curso de ciências contábeis: um estudo em instituições de ensino superior privadas. Advances in Scientific and Applied Accounting, v. 7, p. 407-431, 2014. 
Suzete Antonieta Lizote, Miguel Angel Verdinelli, José Carlos Terres, Elen Sauer Camozzato e Jacqueline dos Santos Seemann

LIZOTE, S. A.; VERDINELLI, M. A.; LANA, J. Satisfação dos alunos dos cursos de pósgraduação lato sensu: um estudo através da modelagem em equações estruturais. In: Anais... XI Colóquio Internacional sobre Gestão Universitária de América do Sul. Florianópolis, 2011.

MARCHETTI, R; PRADO, P. H. M. Um tour pelas medidas de satisfação do consumidor. Revista de Administração de Empresas, v. 41, n. 4, p. 56-67, 2004.

McKENNA, R. Marketing de relacionamento: estratégias bem-sucedidas para a era do cliente. 15 Ed., São Paulo: Campus, 1992.

MEZOMO, J. C. Educação e qualidade total: a escola volta às aulas. Petrópolis: Vozes, 1997.

NAVARRO, M. M.; IGLESIAS, M. P.; TORRES, P. R. A new management element for universities: satisfaction with the offered courses. International Journal of Educational Management, v. 19, n. 6, p. 505-526, 2005.

PALACIO, A. B.; MENESES, G. D.; PÉREZ, P. J. P. The configuration of the university image and its relationship with the satisfaction of students. Journal of Educational Administration, v. 40, n. 5, p. 486-505, 2002.

PASWAN, A. K.; YOUNG, J. A. Student evaluation of instructor: a nomological investigation using structural equation modeling. Journal of Marketing Education, v. 24, n. 3, p. 193-202, 2002.

PORTURAK, M. Private universities service quality and students satisfaction. Global Business and Economics Research Journal, v. 3, n. 2, p. 33-49, 2014.

SCOTT, S. V. The academic as service provider: is the customer 'always right'? Journal of Higher Education Policy and Management, v. 21, n. 2, p. 193-202, 1999.

SENTHILKUMAR, N.; ARULRAJ, A. SQM-HEI: determination of service quality measurement of higher education in India. Journal of Modelling in Management, v.6, n. 1, p. 60-78, 2011.

TABACHNICK, B. G.; FIDEL, L. S. Using Multivariate Statistics. 4 Ed., Boston: Allyn and Bacon, 2001.

TEMIZER, L.; TURKYILMAZ, A. Implementation of student satisfaction index model in higher education institutions. Procedia - Social and Behavioral Sciences, v. 46, p.38023806, 2012.

VERDINELLI, M. A.; SOUZA, M. J. B.; TOMIO, J. L. Análise da relação entre a imagem institucional e a satisfação dos alunos para subsidiar o marketing educacional. In: Anais... IX Colóquio Internacional sobre Gestão Universitária de América do Sul. Florianópolis, 2009.

VIEIRA, K. M.; MILACH, F. T.; HUPPES, R. D. Equações estruturais aplicadas à satisfação dos alunos: um estudo no curso de ciências contábeis da Universidade Federal de Santa Maria. Revista de Contabilidade e Finanças da USP, v. 19, n. 48, p. 65-76, 2008. 
Suzete Antonieta Lizote, Miguel Angel Verdinelli, José Carlos Terres, Elen Sauer Camozzato e Jacqueline dos Santos Seemann

WALTER, S., TONTINI, G., DOMINGUES, M. Identificando Oportunidades de Melhoria em um Curso Superior Através da Análise da Satisfação dos Alunos. In: Anais... XXIX Encontro Nacional de Programas de Pós-Graduação em Administração. Rio de Janeiro: 2005.

WATTY, K. Quality in accounting education: what say the academics? Quality Assurance in Education, v. 13, p. 2, p. 120-131, 2005. 